\title{
The Complexity of Robust Atomic Storage
}

\author{
Dan Dobre \\ TU Darmstadt \\ Darmstadt, Germany \\ dan@cs.tu-darmstadt.de
}

\author{
Rachid Guerraoui \\ EPFL \\ Lausanne, Switzerland \\ rachid.guerraoui@epfl.ch
}

\author{
Matthias Majuntke \\ TU Darmstadt \\ Darmstadt, Germany \\ majuntke@cs.tu- \\ darmstadt.de
}

\author{
Neeraj Suri \\ TU Darmstadt \\ Darmstadt, Germany \\ suri@cs.tu-darmstadt.de
}

\author{
Marko Vukolić \\ EURECOM \\ Sophia-Antipolis, France \\ marko.vukolic@eurecom.fr
}

\begin{abstract}
We study the time-complexity of robust atomic read/write storage from fault-prone storage components in asynchronous message-passing systems. Robustness here means waitfree tolerating the largest possible number $t$ of Byzantine storage component failures (optimal resilience) without relying on data authentication. We show that no single-writer multiple-reader (SWMR) robust atomic storage implementation exists if (a) read operations complete in less than four communication round-trips (rounds), and (b) the timecomplexity of write operations is constant. More precisely, we present two lower bounds. The first is a read lower bound stating that three rounds of communication are necessary to read from a SWMR robust atomic storage. The second is a write lower bound, showing that $\Omega(\log (t))$ write rounds are necessary to read in three rounds from such a storage. Applied to known results, our lower bounds close a fundamental gap: we show that time-optimal robust atomic storage can be obtained using well-known transformations from regular to atomic storage and existing time-optimal regular storage implementations.
\end{abstract}

\section{Categories and Subject Descriptors}

C.2.4 [Computer-Communication Networks]: Distributed Systems; D.4.1 [Operating Systems]: Process Management-concurrency, multiprocessing / multiprogramming / multitasking, synchronization; D.4.5 [Operating Systems]: Reliability_Fault-tolerance

\section{General Terms}

Algorithms, Performance, Reliability, Theory

\footnotetext{
*Dan Dobre is currently also with NEC Laboratories Europe, Kurfürsten-Anlage 36, 69115 Heidelberg, Germany
}

Permission to make digital or hard copies of all or part of this work for personal or classroom use is granted without fee provided that copies are not made or distributed for profit or commercial advantage and that copies bear this notice and the full citation on the first page. To copy otherwise, to republish, to post on servers or to redistribute to lists, requires prior specific permission and/or a fee.

PODC'11, June 6-8, 2011, San Jose, California, USA.

Copyright 2011 ACM 978-1-4503-0719-2/11/06 ...\$10.00.

\section{Keywords}

Lower bounds, Storage emulations, Arbitrary failures, Optimal resilience, Time-complexity

\section{INTRODUCTION}

\subsection{Background}

Variable sharing is critical to modern distributed and concurrent computing. The atomic read/write register abstraction [18] is essential to sharing information in distributed systems; it abstracts away the complexity incurred by concurrent access to shared data by providing processes an illusion of sequential access to data. This abstraction is also referred to as atomic storage, for its importance as a buildingblock in practical distributed storage and file systems (see e.g., $[24,25])$. Besides, its read/write API, despite being very simple, is today the heart of modern "cloud" key-value storage APIs (e.g., [5]).

In this paper, we study atomic storage implementations in asynchronous message-passing systems in which a set of reader and writer processes (clients) share data leveraging a set of storage object processes. We consider fault-tolerant, robust [3] storage implementations characterized by: a) waitfreedom [17], i.e., the fact that read/write operations invoked by correct clients always eventually return, and b) ensuring correctness despite the largest possible number $t$ of object failures (optimal resilience). We allow for the most general type of failures, arbitrary, also called Byzantine [19] failures ${ }^{1}$, without assuming authenticated (also called selfverifying [23]) data to limit the adversary (by relying on e.g., digital signatures).

In this model, we ask a fundamental question: what is the optimal worst-case complexity of robust atomic storage implementations? Our complexity metric is an important one: time-complexity, or latency, measured in number of communication round-trips (or simply rounds) between a client and objects. The relevance of the question we ask extends beyond theoretical. Namely, with the growth in storage outsourcing driven by the advent of cloud computing, the arbitrary failure model becomes increasingly relevant in absence of the full trust in the cloud [6]. In addition, the number of

\footnotetext{
${ }^{1}$ In the Byzantine failure model, optimal resilience corresponds to using $3 t+1$ objects to tolerate $t$ failures [23].
} 
interactions with the remote cloud storage needed to access the data, maps to our latency metric and is often directly associated with the monetary cost; this obviously increases further the practical relevance of the question we ask.

Perhaps surprisingly and despite the wealth of literature exploring latency-optimal storage implementations, this question has not been answered. It is known that the worstcase latency of writing into robust storage is at least 2 rounds [1]. In this paper, we show that the optimal worst-case latency of reading from scalable robust atomic storage is 4 (four) rounds. Here, the notion of scalability captures two basic criteria: a) support for any number of readers, and b) constant write-latency. Our results close a fundamental gap, showing that latency-optimal scalable and robust atomic storage, combining 2-round writes and 4-round reads, can be achieved (in the case of single-writer multi-reader (SWMR) storage) using standard transformations from weaker, regular [18] registers to the atomic ones [4,20].

Our contribution goes through proving two lower bounds. To help fully appreciate our contributions, we first discuss how the scope of this paper fits into related work.

\subsection{Related work}

Several papers have explored the time-complexity metric in the context of a read/write register abstraction. A seminal crash-tolerant robust atomic SWMR register implementation assuming a majority of correct processes was presented in [3]. In [3], all write operations complete in a single round; on the other hand, read operations always take two rounds between a client and objects.

The problem of modifying [3] to enable single round reads was explored in [9], which showed that such fast atomic implementations are possible albeit they come with the price of limited number of readers and suboptimal resilience. Moreover, the reader in [9] needs to write (i.e., modify the objects' state) as dictated by the lower bound of [12] which showed that every atomic read must write into at least $t$ objects. [10] extends the result of [9] to the Byzantine failure model assuming authenticated (i.e., digitally signed) data and established the impossibility of fast crash-tolerant multiwriter multi-reader (MWMR) atomic register implementations. This result is in line with classical MWMR implementations such as [22] that have read/write latency of at least 2 rounds. The limitation on the number of readers of [9], was relaxed in [13], where a crash-tolerant robust SWMR atomic register implementation was presented, in which most of the reads complete in a single round, yet a fraction of reads is permitted to be slow and complete in 2 rounds.

In the Byzantine context, optimizing latency is particularly interesting when data is assumed to be unauthenticated, which we also assume here. [1] showed that any Byzantine-tolerant storage employing at most $4 t$ storage objects has at least some write operation complete in 2 rounds. Moreover, [1] showed a tight lower bound of $t+1$ rounds from reading from robust SWMR safe [18] storage, with the constraint that readers are precluded from writing. However, allowing readers to write helps improve latency as shown in [15], through a 2-round tight lower bound on reading from robust SWMR regular [18] storage. This bound was circumvented in [8], assuming secret values used to detect concurrent operations, where reads are expedited to complete in a single round. However, none of these papers dealt with optimal worst-case latency of reading from robust atomic storage, which is precisely the scope of our paper.

On the other hand, few papers have explored the best-case complexity of Byzantine-tolerant optimally resilient atomic storage. Here, "best-case" encompasses synchrony, no or few object failures and the absence of read/write concurrency. In this context, [14] presented the first robust atomic storage implementation in which both reads and writes are fast in the best-case (i.e., complete in a single round-trip). Furthermore, [16] considered robust atomic storage implementations with the possibility of having fast reads and writes gracefully degrade to 2 or 3 rounds, depending on the size of the available quorum of correct objects. Unlike these papers, we are interested here with the unconditional, worst-case latency of atomic storage.

Finally, the worst-case read latency in existing Byzantinetolerant robust atomic storage implementations for unauthenticated data (e.g., $[2,14,16,23])$ is either unbounded or $\Omega(t)$ rounds at best [2].

\subsection{Contributions}

We present two lower bounds (impossibility results) on time-complexity of reading from robust atomic storage for unauthenticated data, implemented from storage objects prone to Byzantine faults. Together, our lower bounds imply that there is no scalable robust atomic storage implementation in the Byzantine unauthenticated model in which all reads complete in less than 4 rounds.

- The first lower bound, referred to as the read lower bound, demonstrates the impossibility of reading from robust SWMR atomic storage in two rounds. More precisely, we show that if the number of storage objects $S$ is at most $4 t$ and if the number of readers $R$ is greater than 3, then no SWMR atomic implementation may have all reads complete in two rounds.

Our proof scheme resembles that of [9] and relies on sequentially appending reads on a write operation, while progressively deleting the steps of a write and preceding read operations, exploiting asynchrony and possible failures. This deletion ultimately allows reusing readers and reaching an impossibility with as few as $R=4$ readers. As none of these appended operations are concurrent under step contention, the impossibility also holds in the stronger data model of [8], in which the adversary is unable to simulate step contention among operations, making use of secret values.

- Our second lower bound, referred to as the write lower bound, shows that if read operations are required to complete in three communication rounds, then the number of write rounds $k$ is $\Omega(\log (t))$. More precisely, we show that if the number of storage objects is at most $3 t+\left\lfloor t / t_{k}\right\rfloor$ and $R \geq k$, then no implementation of a SWMR atomic storage may have all reads complete in three rounds and all writes in $k \leq\left\lfloor\log \left(\left\lceil\frac{3 t_{k}+1}{2}\right\rceil\right)\right\rfloor$ rounds. In a sense, our lower bound generalizes the write lower bound of [1], which proves our result for the special case of $k=1$.

While using a similar approach, the write lower bound proof is much more involved and differs from our read 
lower bound proof in several key aspects. Due to the additional third read round, read steps cannot be entirely deleted, which prohibits the reuse of readers. Consequently, the number of supported readers $R$ and the number of write rounds $k$ are related $(R \geq k)$. Furthermore, the proof relies on a set of malicious objects that forges critical steps of the write and of prior reads with respect to subsequent reads. This set grows with the number of appended reads, relating the number of faulty objects $t$ and the number of readers (which is at least $k$ ). At the heart of the proof we use a recurrent formula that relates $t$ and $k$, similar to a Fibonacci sequence, which describes the exact relation between the two parameters. In its closed form, the formula transforms to the $\log$ function $(k=\Omega(\log (t)))$.

The rest of the paper is organized as follows. In Section 2 we give our model and definitions. Sections 3 and gives the proof of our read lower bound. ${ }^{2}$ Section 4 gives the proof of our write lower bound. Section 5 concludes the paper by discussing modular implementations that match our lower bounds.

\section{MODEL}

\subsection{Basics}

The distributed system we consider consists of three disjoint sets of processes: a set objects of size $S$ containing processes $\left\{s_{1}, \ldots, s_{S}\right\}$ and representing the base register elements; a singleton writer containing a single process $\{w\}$; and a set readers of size $R$ containing processes $r_{1}, \ldots, r_{R}$. The set clients is the union of the sets writer and readers. We assume that every client may communicate with any process by message passing using point-to-point reliable communication channels. However, objects cannot communicate among each other, nor send messages to clients other than in reply to clients' messages.

Here we define only the notions we use in our proofs; model details can be found in [20]. A distributed algorithm $A$ is a collection of automata [21], where automaton $A_{p}$ is assigned to process $p$. Computation proceeds in steps of $A$; each step is denoted by a pair of process id and a set of messages received in that step $\langle p, M\rangle$ ( $M$ might be $\emptyset)$. A run is an infinite sequence of steps of $A$. A partial run is a finite prefix of some run. A (partial) run $r$ extends some partial run $p r$ if $p r$ is a prefix of $r$. At the end of a partial run, all messages that are sent but not yet received are said to be in transit. In any run, any client can fail by crashing and up to $t$ objects may be malicious faulty, exhibiting arbitrary behavior. The non-faulty objects are also called correct. An algorithm that assumes $S=3 t+1$ is said to be optimally resilient.

\subsection{Atomic Storage}

A register abstraction is a read/write data structure. It provides two operations: write $(v)$, which stores $v$ in the register, and $\operatorname{read}()$, which returns the value from the register. We assume that each client invokes at most one operation at a time (i.e., does not invoke the next operation until it

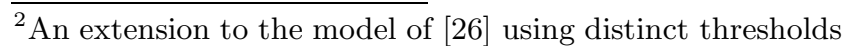
for malicious and crash objects' faults can be found in our full paper [7].
}

receives the response for the current operation). Only readers invoke read operations and only the writer invokes write operations. We further assume that the initial value of a register is a special value $\perp$, which is not a valid input value for a write operation. We say that an operation op is complete in a (partial) run if the run contains a response step for $o p$. In any run, we say that a complete operation $o p_{1}$ precedes operation $o p_{2}$ (or $o p_{2}$ succeeds $o p_{1}$ ) if the response step of $o p 1$ precedes the invocation step of $o p_{2}$ in that run. If neither $o p_{1}$ nor $o p_{2}$ precedes the other, then the operations are said to be concurrent.

An algorithm implements a register if every run of the algorithm satisfies wait-freedom and atomicity properties. Wait-freedom states that if a process invokes an operation, then eventually, unless that process crashes, the operation completes (even if all other client processes have crashed). Here we give a definition of atomicity for the single-writer registers. In the single-writer setting, the writes in a run have a natural ordering which corresponds to their physical order. Denote by $w r_{k}$ the $k^{t h}$ write in a run $(k \geq 1)$, and by $v_{a l}$ the value written by the $k^{t h}$ write. Let $v a l_{0}=\perp$. We say that a partial run satisfies atomicity if the following properties hold: (1) if a read returns $x$ then there is $k$ such that $v a l_{k}=x,(2)$ if a read $r d$ is complete and it succeeds some write $w r_{k}(k \geq 1)$, then $r d$ returns val $_{l}$ such that $l \geq k,(3)$ if a read $r d$ returns $v^{2} l_{k}(k \geq 1)$, then $w r_{k}$ either precedes $r d$ or is concurrent with $r d$, and (4) if some read $r d_{1}$ returns $v a l_{k}(k \geq 0)$ and a read $r d_{2}$ that succeeds $r d_{1}$ returns val $_{l}$, then $l \geq k$.

\section{Time-complexity.}

We measure the time-complexity of an atomic register implementation in terms of communication round-trips (or simply rounds). A round is defined similar to $[9,11,13,22]$ :

DEFINITION 1. Client c performs a communication round during operation op if the following conditions hold:

1. The client $c$ sends messages to all objects. (This is without loss of generality because we can model the fact that messages are not sent to certain objects by having these objects not change their state or reply.)

2. Objects, on receiving such a message, reply to the client before receiving any other messages (as dictated by our model).

3. When the invoking client receives a sufficient number of such replies, the round ( $r n d)$ terminates, and the operation op either completes or moves to the next round.

Note that, since any number of clients can crash, we can construct partial runs in which no client receives any message from any other client. In our proofs in Section 3 and 4 we focus, without loss of generality, on such partial runs.

Since up to $t$ objects might be faulty, ideally, in every round $r n d$ the invoking client can only wait for reply messages from correct objects (at least $S-t$ ). In fact, we require that if in a partial run $p r$, a round $r n d$ terminates without the reply from some object $s_{i}$, then either (a) $s_{i}$ is faulty or (b) there is partial run $p r^{\prime}$ indistinguishable from $p r$, and in which $s_{i}$ is faulty.

Each round attempts to invoke operations on all objects. If on some correct object $s_{i}$ there is a pending invocation (of an earlier round), then the new invocation awaits the 
completion of the pending one. Note that this is equivalent to the round model of [1].

\section{THE READ LOWER BOUND}

In this section we prove the following proposition.

Proposition 1. : If $S \leq 4 t$ and $R>3$, then no read implementation I of a multi-reader ( $S W M R$ ) atomic register exists that completes in two rounds.

\section{Overview.}

The idea behind the proof is to start with a complete write that writes 1 into the storage, after which a complete read is appended. By atomicity, the read returns 1. Then, further reads by distinct readers are appended one after the other such that the last appended read returns 1 . At the same time, steps of the write and the previous reads are progressively deleted. After appending the fourth read, the final round of the write is deleted from the storage. Moreover, similar to a circular buffer, all steps of the first read are erased, and the read ca be "recycled". By atomicity, the last appended read returns 1 . The next iteration starts by reusing the first read, which in turn frees the second read. The proof proceeds through a sequence of such iterations. In each iteration, the last appended read frees the first appended read, and deletes another round of the write. After the last iteration, all steps of the write are deleted, meaning that no write is invoked. However, the last appended read returns 1, violating atomicity.

\section{Preliminaries.}

In the proof $w$ denotes the writer, $r_{i}$ for $1 \leq i \leq 4$ denote the readers, and $s_{i}$ for $1 \leq i \leq S$ denote objects. Suppose by contradiction that $R=4$ and there is an atomic register implementation $I$ that uses at most $4 t$ objects, such that in every partial run of $I$ every read operation completes in two rounds.

We partition the set objects into four disjoint subsets (which we call blocks), denoted $B_{1}, B_{2}, B_{3}$ and $B_{4}$. Blocks $B_{1}, B_{2}$ and $B_{3}$ are of size exactly $t \geq 1$ and the size of $B_{4}$ is at least 1 and at most $t$. We refer to the initial state of every correct block $B_{j}$ as $\sigma_{0}^{j}$. For simplicity we simply write $\sigma_{0}$, where the block name is implicit.

We say that a round $r n d$ of an operation op skips a set of blocks $B S$ in a partial run, (where $B S \subseteq\left\{B_{1}, \ldots, B_{4}\right\}$ ), if (1) no object in any block $B L \in B S$ receives any message in round $r n d$ from $o p$ in that partial run; (2) all other objects receive all messages in round $r n d$ from $o p$ and reply to the messages, and (3) in case round $r n d$ is terminated, the invoking client has received all these reply messages or, in case $r n d$ is not terminated, all these reply messages are in transit. We say that an operation op skips a set of blocks $B S$ in a partial run if every round of $o p$ skips $B S$.

To show a contradiction, we construct a partial run of the implementation $I$ that violates atomicity: a partial run of $I$ in which no value is ever written and some read returns 1.

\section{Partial writes.}

Throughout the proof there is only one write operation write(1) by $w$ that writes value 1 . Consider a partial run $w r$ in which $w$ completes write(1) on the register and let $k$ be the number of rounds invoked by $w$ in $w r$. We denote the state of every correct block $B_{j}$ after it has replied to the messages of the write during round 1 to $i$ where $1 \leq i \leq k$ as $\sigma_{i}$, where $j$ is again implicit. The write operation skips blocks $B_{4}$. We define a series of partial runs containing an incomplete write(1) invocation, each being a prefix of $w r$. For $1 \leq i \leq k$ and $1 \leq j \leq 4$, we define $w r_{j}^{i}$ as the partial run in which (1) rounds 1 to $i-1$ are terminated and skip $B_{4} ;(2)$ round $i$ is not terminated and skips all blocks $\left\{B_{l} \mid 1 \leq l \leq j-1\right\} \cup\left\{B_{4}\right\}$, and (3) all objects are correct. We make two observations: (1) partial run $w r_{1}^{k}$ differs from $w r$ only at $w$ and (2) partial run $w r_{4}^{1}$ differs from a run in which write(1) is never invoked only at $w$.

\section{Block diagrams.}

We illustrate the proof in Figure 1 (a)-(n). We depict a round $r n d$ of an operation op through a set of rectangles arranged in a single column. In the column corresponding to some round $r n d$ of $o p$ we draw a rectangle in a given row, if all objects in the corresponding block $B L$ have received the message from the client in round $r n d$ of $o p$ and have sent reply messages, i.e., if round $r n d$ of $o p$ does not skip $B L$. We write "@" in the row corresponding to $B L$ iff $B L$ is malicious.

\section{Appending reads.}

Partial run $p r_{1}$ extends $w r$ by appending a complete read $r d_{1}$ by $r_{1}$ that skips $B_{2}$ in round one and $B_{1}$ in round two (see Figure 1 (a)). Note that when the second round is started, there is a pending first round invocation on $B_{2}$. Therefore in the second round, $r d_{1}$ waits for both first and second round replies from $B_{2}$. For ease of presentation, the late first round replies are not illustrated.

In $p r_{1}$, all objects in block $B_{1}$ are malicious, and forge their state to $\sigma_{k-1}$ before replying to $r d_{1}$. By atomicity $r d_{1}$ returns 1. Observe that $r_{1}$ cannot distinguish $p r_{1}$ from some partial run $\Delta p r_{1}$ that extends $w r_{2}^{k}$ by appending $r d_{1}$, and where all objects are correct (see Figure 1 (b)). Note that $\Delta p r_{1}$ is obtained from $p r_{1}$ by deleting the crossed steps.

Partial run $p r_{2}$ extends $\Delta p r_{1}$ by appending a complete read $r d_{2}$ by $r_{2}$ that skips $B_{3}$ and $B_{2}$ in round one and two respectively (see Figure 1 (c)). In $p r_{2}$, all objects in block $B_{2}$ are malicious, and forge their state to $\sigma_{k-1}$ before replying to $r d_{2}$. By atomicity $r d_{2}$ returns 1 . Observe that $r_{2}$ cannot distinguish $p r_{2}$ from some partial run $\Delta p r_{2}$, that extends $w r_{3}^{k}$ by appending an incomplete $r d_{1}$ and a complete $r d_{2}$, and where all objects are correct (Figure $1(\mathrm{~d})) . \Delta p r_{2}$ is obtained from $p r_{2}$ by deleting the crossed steps.

Partial run $p r_{3}$ extends $\Delta p r_{2}$ by appending a complete read $r d_{3}$ by $r_{3}$ that skips $B_{4}$ in round one and $B_{3}$ in round two (Figure $1(\mathrm{e})$ ). In $p r_{3}$, all objects in block $B_{3}$ are malicious, and forge their state to $\sigma_{k-1}$ before replying to $r d_{3}$. By atomicity $r d_{3}$ returns 1 . Let $\sigma_{1}^{r}$ denote the state of the objects in block $B_{4}$ in run $p r_{3}$ before replying to $r d_{2}$. Observe that $r_{3}$ cannot distinguish $p r_{3}$ from some partial run $\Delta p r_{3}$, that extends $w r_{4}^{k}$ by appending incomplete reads $r d_{1}$ and $r d_{2}$ and a complete read $r d_{3}$ and in which (1) all objects in $B_{4}$ are malicious and (2) they forge their state to $\sigma_{1}^{r}$ before replying to $r d_{2}$ (Figure 1 (f)).

Note that in $p r_{3}, r d_{3}$ completes the second round based on replies from all correct objects, and similarly in $\Delta p r_{3}$, the first round misses replies only from faulty objects. Since $r_{3}$ cannot distinguish $p r_{3}$ and $\Delta p r_{3}$, it cannot wait for additional replies (in any of the two runs). 


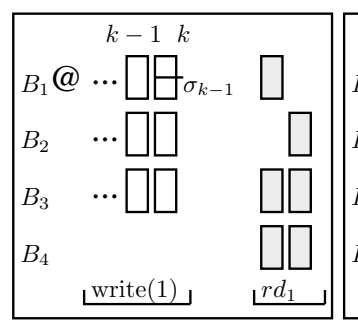

(a) $p r_{1}$ extends $w r$

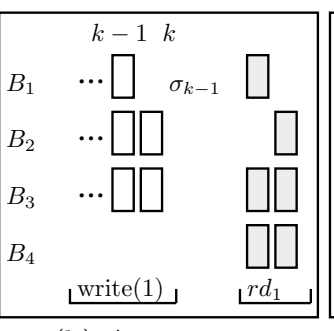

(b) $\Delta p r_{1} \sim p r_{1}$

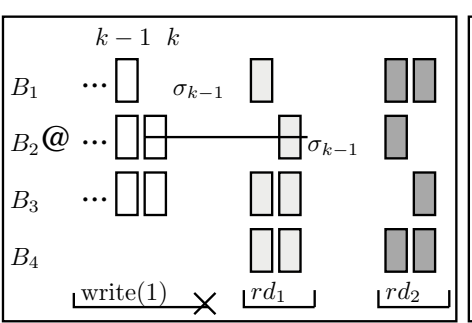

(c) $p r_{2}$ extends $\Delta p r_{1}$

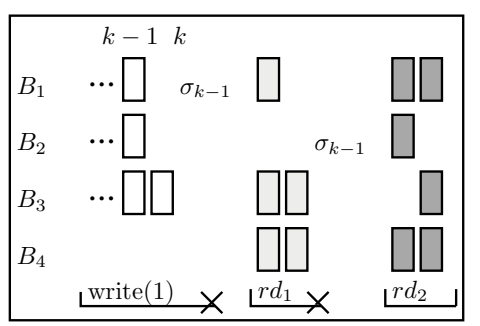

(d) $\Delta p r_{2} \sim p r_{2}$

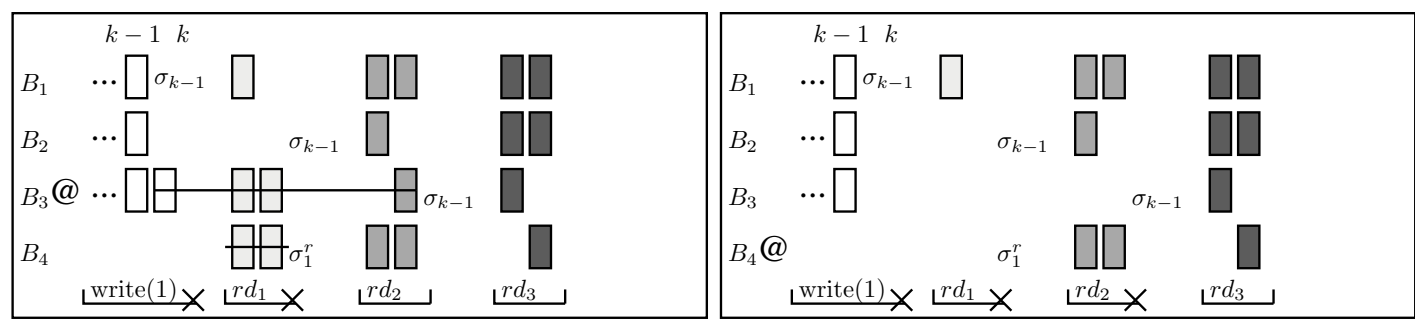

(e) $p r_{3}$ extends $\Delta p r_{2}$

(f) $\Delta p r_{3} \sim p r_{3}$

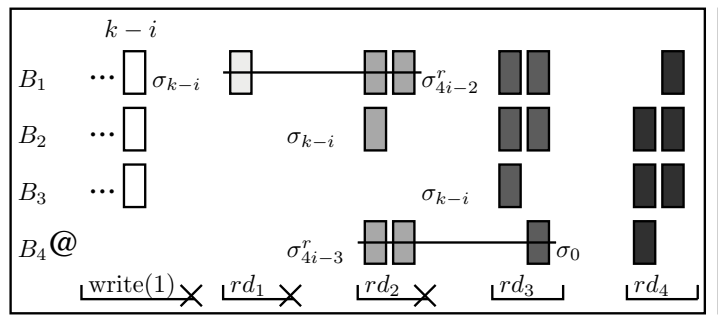

(g) $p r_{4 i}$ extends $\Delta p r_{4 i-1}$

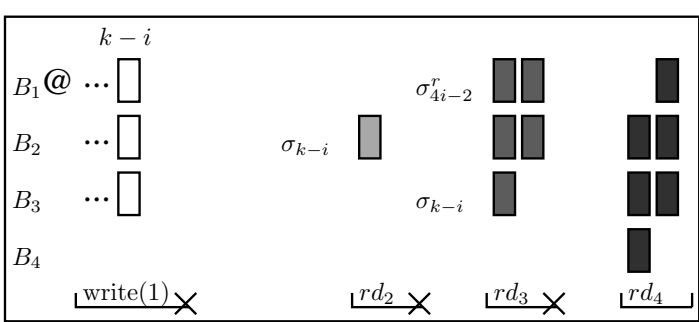

(h) $\Delta p r_{4 i} \sim p r_{4 i}$

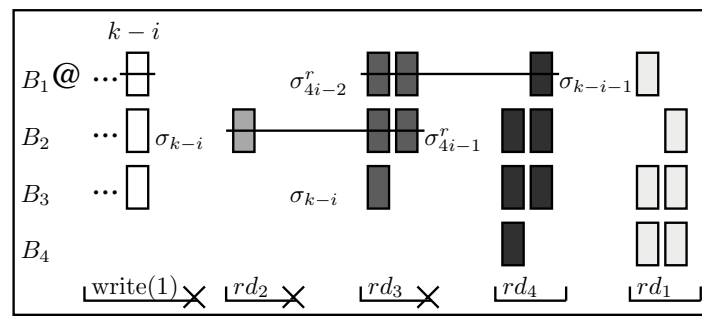

(i) $p r_{4 i+1}$ extends $\Delta p r_{4 i}$

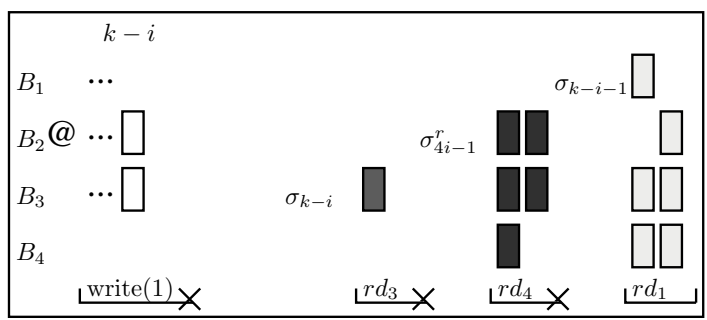

(j) $\Delta p r_{4 i+1} \sim p r_{4 i+1}$

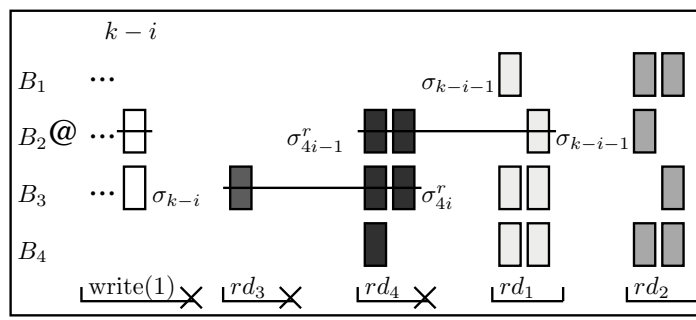

(k) $p r_{4 i+2}$ extends $\Delta p r_{4 i+1}$

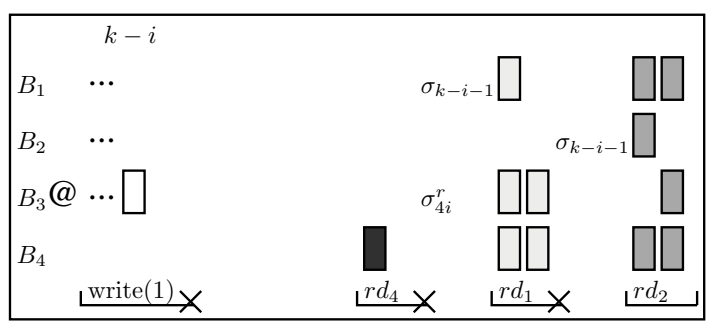

(l) $\Delta p r_{4 i+2} \sim p r_{4 i+2}$

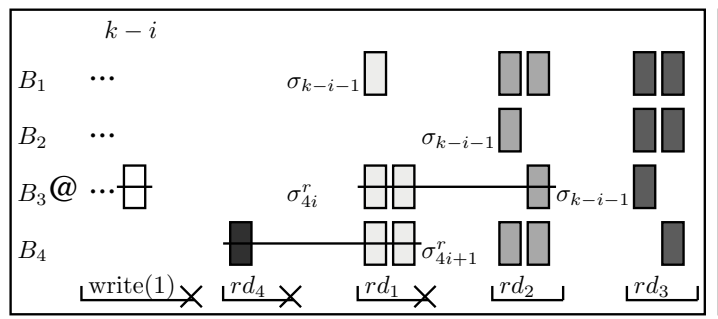

(m) $p r_{4 i+3}$ extends $\Delta p r_{4 i+2}$

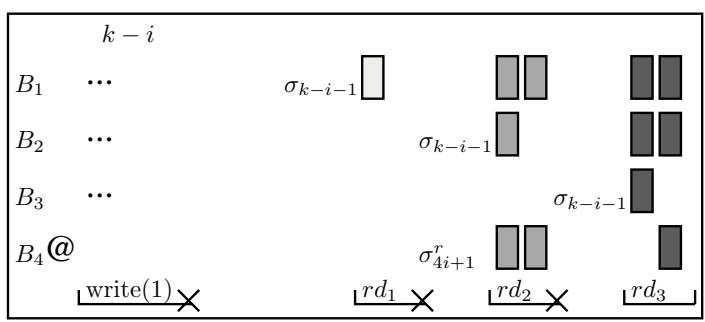

(n) $\Delta p r_{4 i+3} \sim p r_{4 i+3}$

Figure 1: Illustration of the runs used in the proof of Proposition $1(1 \leq i \leq k-1)$ 
Partial run $p r_{4}$ (illustrated in Figure $1(\mathrm{~g})$ ) extends $\Delta p r_{3}$ by appending a complete read $r d_{4}$ by $r_{4}$ that skips $B_{1}$ in round one and $B_{4}$ in round two. In $p r_{4}$, all objects in block $B_{4}$ are malicious and forge their state (1) to $\sigma_{1}^{r}$ before replying to $r d_{2}$ and (2) to $\sigma_{0}$ before replying to $r d_{4}$. By atomicity $r d_{4}$ returns 1 . Let $\sigma_{2}^{r}$ denote the state of the objects in block $B_{1}$ before replying to $r d_{3}$. Observe that $r_{4}$ cannot distinguish $p r_{4}$ from some partial run $\Delta p r_{4}$, that extends $w r_{1}^{k-1}$ by appending incomplete reads $r d_{2}, r d_{3}$ and a complete read $r d_{4}$, and in which (1) all objects in $B_{1}$ are malicious and (2) they forge their state to $\sigma_{2}^{r}$ before replying to $r d_{3}$ (Figure 1 (h)). Note that in partial run $p r_{4}, r d_{4}$ receives second round replies from all correct objects. Similarly in $\Delta p r_{4}, r d_{4}$ receives first round replies from all objects except the faulty ones. Since $r_{4}$ cannot distinguish $p r_{4}$ and $\Delta p r_{4}, r d_{4}$ cannot wait for additional replies without violating termination.

After appending $r d_{4}$ and constructing $\Delta p r_{4}$ by deleting all steps from $p r_{4}$ which are not visible to $r d_{4}$, we notice that we have erased all steps in column $k$ of write(1) as well as, deleted all steps of $r d_{1}$. Thus, we can recycle $r_{1}$ by appending $r d_{1}$ again and start deleting the steps in column $k-1$.

Starting from $\Delta p r_{4}$ we iteratively define the following partial runs for $1 \leq i \leq k-1$ and $1 \leq j \leq 4$ (see Figure 1 (g)(n)). Partial run $p r_{4 i+(j \bmod 4)}$ extends $\Delta p r_{4 i+(j \bmod 4)-1}$ by appending $r d_{j}$. In $p r_{4 i+(j \bmod 4)}$, all objects in block $B_{j}$ are malicious and they forge their state $(1)$ to $\sigma_{4 i+(j \bmod 4)-3}^{r}$ before replying to $r d_{j-2}{ }^{3}$ and $(2)$ to $\sigma_{((j \bmod 4) / j)(k-i-1)}$ before replying to $r d_{j}$. Let $\sigma_{4 i+(j \bmod 4)-2)}^{r}$ denote the state of the objects in block $B_{(j \bmod 4)+1}$ before replying to $r d_{j-1}$. Observe that $r_{j}$ cannot distinguish $p r_{4 i+(j \bmod 4)}$ from some partial run $\Delta p r_{4 i+(j \bmod 4)}$, that extends $w r_{(j \bmod 4)+1}^{k-i}$ by appending incomplete reads $r d_{j-2}$ and $r d_{j-1}$ and a complete read $r d_{j}$, and in which (1) all objects in $B_{(j \bmod 4)+1}$ are malicious, and (2) they forge their state to $\sigma_{4 i+(j \bmod 4)-2}^{r}$ before replying to $r d_{j-1}$ (Figure $\left.1(\mathrm{~h}),(\mathrm{j}),(\mathrm{l}),(\mathrm{n})\right)$. In run $\Delta p r_{4 i+(j \bmod 4)}$ and $p r_{4 i+(j \bmod 4)}, r d_{j}$ receives first and second round replies from all correct objects respectively. As $r_{j}$ cannot distinguish $\Delta p r_{4 i+(j \bmod 4)}$ and $p r_{4 i+(j \bmod 4)}$, $r d_{j}$ cannot wait for additional replies without blocking.

Read $r d_{4}$ in $\Delta p r_{4}$ returns 1 . Since $p r_{5}$ extends $\Delta p r_{4}$ by appending $r d_{1}$, by atomicity, $r d_{1}$ in $p r_{5}$ returns 1 . However, as $r_{1}$ cannot distinguish $p r_{5}$ from $\Delta p r_{5}, r d_{1}$ in $\Delta p r_{5}$ returns 1. In general, since $p r_{4 i+(j \bmod 4)}$ extends $\Delta p r_{4 i+(j \bmod 4)-1}$ by appending $r d_{j}$ (for $1 \leq i \leq k-1$ and $1 \leq j \leq 4$ ), and $r_{j}$ cannot distinguish $p r_{4 i+(j \bmod 4)}$ from $\Delta p r_{4 i+(j \bmod 4)}$, it follows by induction that $r d_{j}$ in $\Delta p r_{4 i+(j \bmod 4)}$ returns 1 . In particular, $r d_{3}$ reads 1 in $\Delta p r_{4 k-1}$. By our construction, $\Delta p r_{4 k-1}$ extends $w r_{4}^{1}$ and $w r_{4}^{1}$ is indistinguishable from a run in which write(1) is never invoked. Hence, $r d_{3}$ returns 1 even if no write is invoked, violating atomicity.

\section{THE WRITE LOWER BOUND}

In this section we prove the following proposition.

Proposition 2. : If $S \leq 3 t+\left\lfloor t / t_{k}\right\rfloor$ and every read completes in three rounds then no write implementation $I$ of a multi-reader atomic register exists that completes in $\min \left\{R,\left\lfloor\log \left(\left\lceil\left(3 t_{k}+1\right) / 2\right\rceil\right)\right\rfloor\right\}$ rounds.

\footnotetext{
${ }^{3}$ By $r d_{j-c}$, we denote the $c^{\text {th }}$ last read prior to $r d_{j}$. Formally, $\left.r d_{4-((c-j)} \bmod 4\right)$.
}

We first prove the following key lemma. In the effort of making its involved proof easier to follow we first proceed through a careful proof setup that we found worthwhile. To further help follow the proof, we also visualize runs we use in the proof in Figure 2.

LemMA 1. Let $k \geq 1, t_{-1}=t_{0}=0$ and $t_{k}=t_{k-1}+$ $2 t_{k-2}+1$. There is no implementation $I$ of a $k$-reader atomic storage with $3 t_{k}+1$ objects and $t_{k}$ faults such that the write completes in $k$ rounds and the read completes in three rounds.

\section{Overview.}

The idea of the proof consists of having a complete write that writes 1 into the storage, after which we append a sequence of read operations. We use patterns of concurrency and failures such that the first read in the sequence cannot distinguish if it overlaps with or succeeds the write. In each case, by atomicity, it must return 1 . For each of the following reads we use the same indistinguishability argument such that the last read in the sequence cannot tell if it is concurrent with or follows after the preceding read. In both cases, by atomicity, the last appended read has to return 1.

To derive a contradiction, for each appended read, we progressively delete one of the $k$ rounds of the write and rounds of the previous reads. As a consequence, the $k^{\text {th }}$ appended read returns 1 in a run in which 1 is never written. To reduce the information about the write propagated via the $3^{\text {rd }}$ read round, as more reads are appended, a monotonically increasing set of base object present forged information. Consecutive read operations increasingly skip these faulty objects together and therefore overlap in an increasing number of correct objects. If two rounds of consecutive reads, both skip the same set of $x$ faulty objects, then they overlap in $2 x+1$ correct objects. To derive a contradiction for the $k^{\text {th }}$ appended read, the set of faulty objects consists of those needed to derive a contradiction for the $k-1^{\text {st }}$ read plus the $2 x+1$ objects in the intersection of the $3^{\text {rd }}$ round of the $k-1^{\text {st }}$ read and the first round of the $k^{\text {th }}$ read. In fact, $x$ equals the number of faulty objects needed to derive a contraction for the $k-2^{\text {nd }}$ read. Together, this leads to the recursive formula used throughout the proof.

\section{Preliminaries.}

Recall that $w$ denotes the writer, $r_{i}$ for $1 \leq i \leq k$ denote the readers, and $s_{i}$ for $1 \leq i \leq S$ denote the objects. The initial value of the register is $\perp$. In the proof, there is only one write operation write(1) by $w$ that writes value 1 . We know from [1] that the lemma is true for $k=1$; hence, we assume $k \geq 2$. Suppose by contradiction that there is an implementation $I$ that uses at most $3 t_{k}+1$ objects, such that in every partial run of $I$ every write (resp., read) completes in $k$ (resp. 3) rounds.

We partition the set objects into $2 k+2$ distinct blocks, $B_{0}, \ldots, B_{k+1}$ and $C_{1}, \ldots, C_{k}$ such that $\left|\bigcup_{j=0}^{k+1} B_{j}\right|=2 t_{k}+1$ and $\left|\bigcup_{j=1}^{k} C_{j}\right|=t_{k}$. Block $B_{0}$ contains a single object. For $1 \leq l \leq k$, the size of $B_{l}$ is $t_{l}-t_{l-2}$ and the size of $B_{k+1}$ is $2 t_{k}+1-\left|\bigcup_{j=0}^{k} B_{j}\right|=t_{k}-t_{k-1}$. For $1 \leq l \leq k-1$, the size of $C_{l}$ is $t_{l-1}-t_{l-2}$ and the size of $C_{k}$ is $t_{k}-\left|\bigcup_{j=1}^{k-1} C_{j}\right|=$ $t_{k}-t_{k-2}$. It is important to note that $C_{1}$ is empty. Towards a uniform presentation of the result, we will refer to $C_{1}$ 
wherever appropriate. Also, we use the abbreviation $B L_{i, j}$ to denote the set $\left\{B L_{i}, B L_{j}\right\}$, for some $B L \in\{B, C\}$.

We also define three sets of blocks called superblocks: the "malicious" superblock $\mathcal{M}_{l}$, the "parity" superblock $\mathcal{P}_{l}$ and the "correct" superblock $\mathcal{C}_{l}$. Superblock $\mathcal{M}_{l}$ contains all blocks with index at most $l$. Formally, for $-1 \leq l \leq k-1$ we define $\mathcal{M}_{l}:=\left\{B_{j} \mid 0 \leq j \leq l\right\} \cup\left\{C_{j} \mid 1 \leq j \leq l\right\}$. For instance, $\mathcal{M}_{-1}=\emptyset$ and $\mathcal{M}_{2}=\left\{B_{0}, B_{1}, C_{1}, C_{2}\right\}$. Superblock $\mathcal{P}_{l}$ contains all blocks $B_{j}$ with index $j \geq l \geq 1$ such that $j$ and $l$ have the same parity. More formally, for $1 \leq l \leq k$, we define $\mathcal{P}_{l}:=\left\{B_{j} \mid l \leq j \leq k+1 \wedge j \equiv(l \bmod 2)\right\}$. For instance, if $k$ is even then $\overline{\mathcal{P}}_{1}=\left\{B_{1}, B_{3}, \ldots, B_{k-1}, B_{k+1}\right\}$ and $\mathcal{P}_{2}=\left\{B_{2}, B_{4}, \ldots, B_{k-2}, B_{k}\right\}$. Finally, superblock $\mathcal{C}_{l}:=$ $\left\{C_{j} \mid l \leq j \leq k\right\}$.

Given the size of the individual blocks, we can determine the cardinality of the union of all elements of a superblock. Namely, if $\mathcal{S} \in\left\{\mathcal{M}_{l}, \mathcal{P}_{l}, \mathcal{C}_{l}\right\}$, then we define the union of its elements as $\bigcup \mathcal{S}=\{s \in B L \mid B L \in \mathcal{S}\}$. Having in mind that $t_{k}=t_{k-1}+2 t_{k-2}+1$ (Def.) and $t_{-1}=t_{0}=0$, we have:

$$
\begin{gathered}
\left|\bigcup \mathcal{M}_{l}\right|=t_{l}+2 t_{l-1}+1 \stackrel{(\text { Def. })}{=} t_{l+1} \quad \text { for } 0 \leq l \leq k-1 \\
\left|\bigcup \mathcal{P}_{l}\right|=t_{k}-t_{l-2} \quad \text { for } \quad 1 \leq l \leq k+1 \\
\left|\bigcup \mathcal{C}_{l}\right|=t_{k}-t_{l-2} \quad \text { for } \quad 1 \leq l \leq k
\end{gathered}
$$

\section{Block diagrams.}

Figure 2 illustrates the proof for $R=k=4$. Reader $r_{i}$ invokes read $r d_{l}, 1 \leq l \leq k$. In the column corresponding to some round $r n d$ of $o p$ we draw a rectangle in a given row, iff round $r n d$ of $o p$ does not skip ${ }^{4}$ the corresponding block $B L$. We write "@" in the row of $B L$ iff $B L$ is malicious.

\section{Read patterns.}

We first characterize a complete read $r d_{l}$ for $1 \leq l \leq k-1$. A complete $r d_{l}$ skips (1) $\mathcal{M}_{l-2} \cup \mathcal{P}_{l+1}$ in round one and two, and (2) $\mathcal{M}_{l-2} \cup \mathcal{C}_{l+1}$ in round three. Read $r d_{k}$ skips $\mathcal{M}_{k-2} \cup \mathcal{P}_{k+1}$. Observe that by equations (1), (2) and (3), a read skips exactly $t_{k}$ objects in each round.

Consider the example in Figure 2. Complete reads $r d_{1}$, $r d_{2}$ and $r d_{3}$ skip (respectively): (1) $\left\{B_{2,4}\right\},\left\{B_{0}\right\} \cup\left\{B_{3,5}\right\}$ and $\left\{B_{0,1}\right\} \cup\left\{B_{4}\right\}$ in rounds one and two, and (2) $\left\{C_{2,3,4}\right\}$, $\left\{B_{0}\right\} \cup\left\{C_{3,4}\right\}$ and $\left\{B_{0,1}\right\} \cup\left\{C_{4}\right\}$ in round three. Read $r d_{4}$ skips $\left\{B_{0,1,2}, C_{2}\right\} \cup\left\{B_{5}\right\}$.

We further define three types of incomplete reads inc1, inc2 and inc3, depending on the read's progress. For $1 \leq l \leq$ $k$, read $r d_{l}$ is of type inc1 if the first round is not terminated and skips all blocks except $\mathcal{P}_{l}$. For $1 \leq l \leq k-1$, read $r d_{l}$ is of type (1) inc2 if the first round is terminated, and the second round is not terminated and skips all blocks except $\mathcal{C}_{l}$, and (2) inc3 if the second round is terminated and the third round is not terminated and skips $\mathcal{M}_{l-2} \cup \mathcal{C}_{l+1} \cup \mathcal{P}_{l+1}$.

Consider our example in Figure 2 (c) that illustrates partial run $\Delta p r_{2}$ (after deleting the crossed out steps). Observe that (1) $r d_{2}$ is incomplete of type inc3 (its third round skips $\left.\left\{B_{0}\right\} \cup\left\{C_{3,4}\right\} \cup\left\{B_{3,5}\right\}\right),(2) r d_{1}$ is incomplete of type inc2 (its second round skips all blocks except $\left\{C_{2,3,4}\right\}$ ) and (3) $r d_{3}$ (resp., $r d_{4}$ ) is incomplete of type $i n c 1$; its first round skips all blocks except $\left\{B_{3,5}\right\}$ (resp., $\left\{B_{4}\right\}$ ).

\footnotetext{
${ }^{4}$ The definition of skipping extends here from Sec. 3 .
}

Towards a contradiction, we construct a partial run of the atomic register implementation $I$ that violates atomicity. More specifically, we exhibit a partial run in which some read returns a value that was never written.

\section{Initialization.}

Consider a partial run $p_{\text {init }}$ in which (1) all blocks are correct and (2) $p r_{\text {init }}$ extends the empty run by appending incomplete reads $r d_{l}$ by $r_{l}$ of type inc1, for $1 \leq l \leq k$, one after the other. In $p r_{\text {init }}$, there is no write operation. We refer to the state of each correct block $B L \in \mathcal{P}_{l}$ after replying to $r d_{l}$ as $\sigma_{0}^{l}$. Thus, the state of $B_{l}$ at the end of $p r_{\text {init }}$ corresponds to $\sigma_{0}^{l}$ for $1 \leq l \leq k$. Further, $B_{k+1}$ is in state $\sigma_{0}^{k-1}$. To see why, note that $\bar{B}_{k-1}$ and $B_{k+1}$ have the same parity and there are only $k$ reads.

Consider our example Figure 2 (a). At the end of $\mathrm{pr}_{\text {init }}$, block $B_{1}$ (resp., $B_{2} ; B_{3,5} ; B_{4}$ ) replied to $r d_{1}$ (resp., $r d_{2} ; r d_{1}$ and $r d_{3} ; r d_{2}$ and $\left.r d_{4}\right)$; thus, at the end of the run its state is $\sigma_{0}^{1}$ (resp., $\sigma_{0}^{2} ; \sigma_{0}^{3} ; \sigma_{0}^{4}$ ).

\section{Partial writes.}

We extend $p r_{\text {init }}$ to a partial run $w r^{k}$ by appending a complete write(1) that completes in $k$ rounds and skips superblock $\mathcal{C}_{1}$. Moreover, we define a series of partial runs each being a prefix of $w r^{k}$. For $1 \leq i \leq k$, let $w r^{k-i}$ be the partial run which extends $p r_{\text {init }}$ by appending an incomplete write(1) such that (i) round 1 to $k-i$ are terminated and (ii) round $k-i+1$ is not terminated and skips $\mathcal{C}_{1}$ and all $B_{j}$ 's such that $j>0$ and $i$ and $j$ have the same parity, i.e., $\mathcal{C}_{1} \cup \mathcal{P}_{2-(i \bmod 2)}$ (Fig. 2 (a) and (c)). We refer to the state of the blocks $B_{l} \in \mathcal{P}_{2-(i \bmod 2)}$ at the end of $w r^{k-i}$ as $\sigma_{k-i}^{l}$ for $1 \leq l \leq k$. If $B_{k+1} \in \mathcal{P}_{2-(i \bmod 2)}$, then we refer to its state at the end of $w r^{k-i}$ as $\sigma_{k-i}^{k-1}$. Note here that $\sigma_{k-i}^{l}$ results from $\sigma_{0}^{l}$ by appending $k-i$ rounds of the write. When the context is clear, for simplicity we refer to these states using the implicit notation $\sigma_{k-1}^{*}$. Finally, we refer to the state of $B_{0}$ at the end of runs $w r^{k}$ and $w r^{k-1}$ as $\sigma_{k}$.

We refer to our example in Figure 2 (a),(c),(e) and (g) for illustrations of the runs $w r^{3}$ to $w r^{0}$ and the corresponding states. For instance Figure 2 (a), illustrates $w r^{3}$ as an extension of $p r_{\text {init }}$. The states of the blocks $B_{0}, B_{1}$ and $B_{3,5}$ at the end of $w r^{3}$ are $\sigma_{4}$ (4 rounds of write), $\sigma_{3}^{1}$ and $\sigma_{3}^{3}$ (3 rounds of write).

\section{Appending Reads.}

Partial run $p r_{1}$ extends $w r^{k-1}$ by appending the missing steps of a complete read $r d_{1}$. In $p r_{1}$ all objects are correct and thus $r d_{1}$ receives replies from $S-t_{k}$ correct objects. After receiving the third round replies, $r d_{1}$ completes and returns value $x$. We now show that $x=1$. We define a partial run @ $p r_{0}$, (Fig. 2(b)) which is identical to $w r^{k}$ except that in @ $p_{0}$ (1) no read by $r_{1}$ occurs and (2) superblock $\mathcal{P}_{1}$ is malicious and mimics the occurrence of $r d_{1}$ by forging its initial state to $\sigma_{0}^{1}$. By equation (1), the malicious objects in @ $p r_{0}$ amount to $t_{k}$. Partial run $p r_{1}^{C}$ (Fig. 2(b)) is defined as an extension of @pr 0 by appending a complete read $r d_{1}$. Read $r d_{1}$ cannot distinguish $p r_{1}^{C}$ from $p r_{1}$ because $\mathcal{P}_{1}$, which is malicious in $p r_{1}^{C}$, mimics $p r_{1}$. Specifically, $\mathcal{P}_{1}$ forges its state to $\sigma_{0}$ before replying to $r d_{1}$ 's first round, and then to $\sigma_{k-1}^{*}$ before replying to $r d_{1}$ 's second round. In $p r_{1}^{C}$, by atomicity $r d_{1}$ returns 1 . Since $p r_{1}^{C}$ and $p r_{1}$ are indistinguishable to reader $r_{1}, x=1$. 


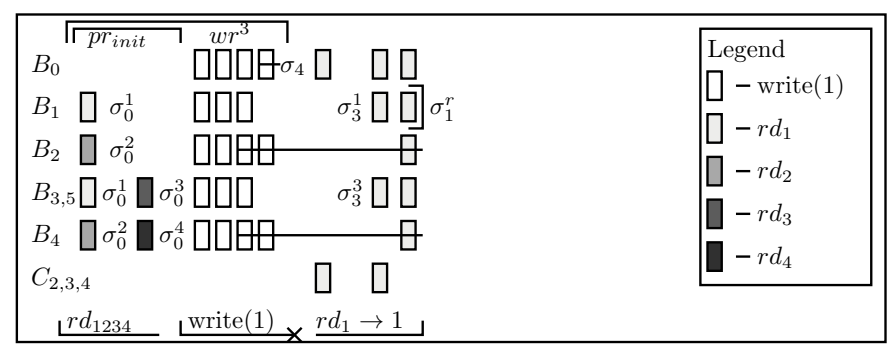

(a) $p r_{1}$ extends $w r^{3}$ ( $\Delta p r_{1}$ from $p r_{1}$ by deleting crossed steps)

\begin{tabular}{|c|c|}
\hline & 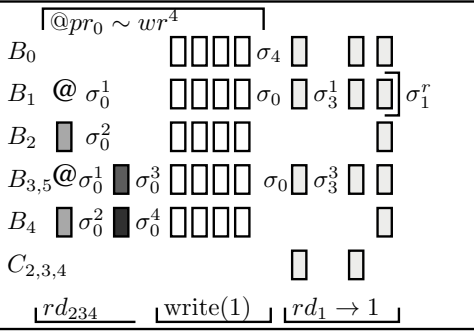 \\
\hline
\end{tabular}

(b) $p r_{1}^{C}$ (extends @ $p r_{0} \sim w r^{k}$ )

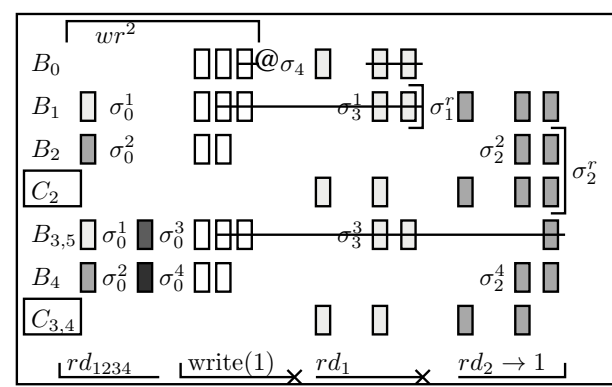

(c) $p r_{2}$ extends $\Delta p r_{1}$ ( $\Delta p r_{2}$ from $p r_{2}$ by deleting crossed steps)

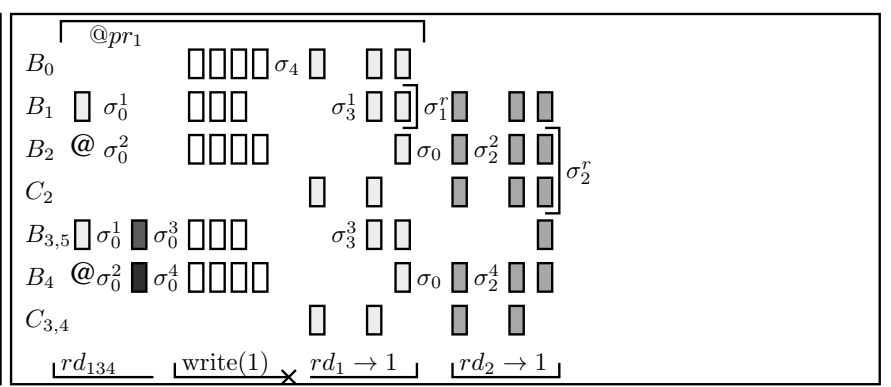

(d) $p r_{2}^{C}$ (extends @ $p r_{1} \sim p r_{1}$ )

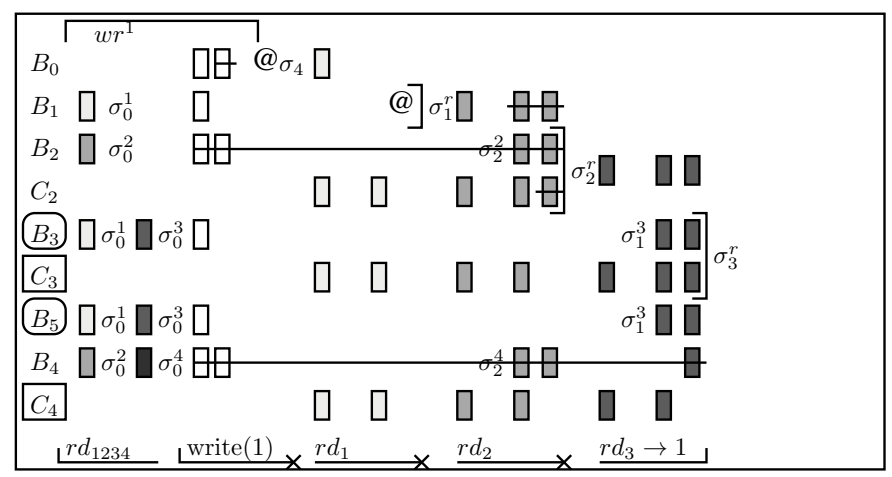

(e) $p r_{3}$ extends $\Delta p r_{2}$ ( $\Delta p r_{3}$ from $p r_{3}$ by deleting crossed steps)

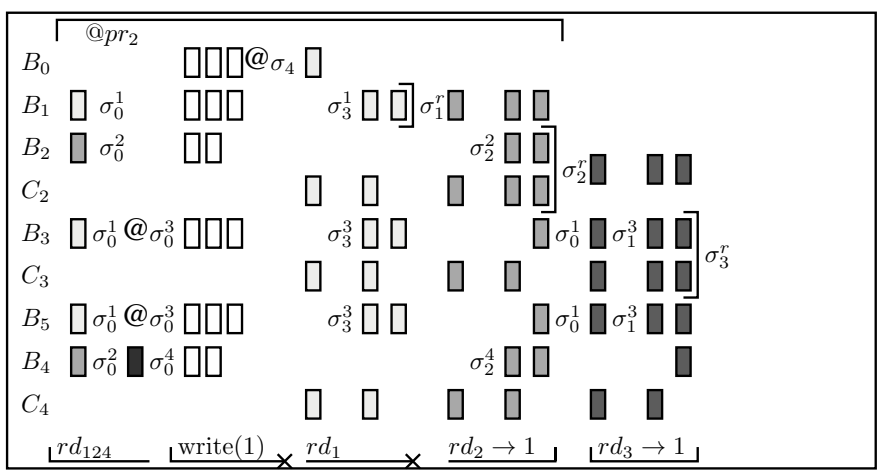

(f) $p r_{3}^{C}$ (extends @ $p r_{2} \sim p r_{2}$ )

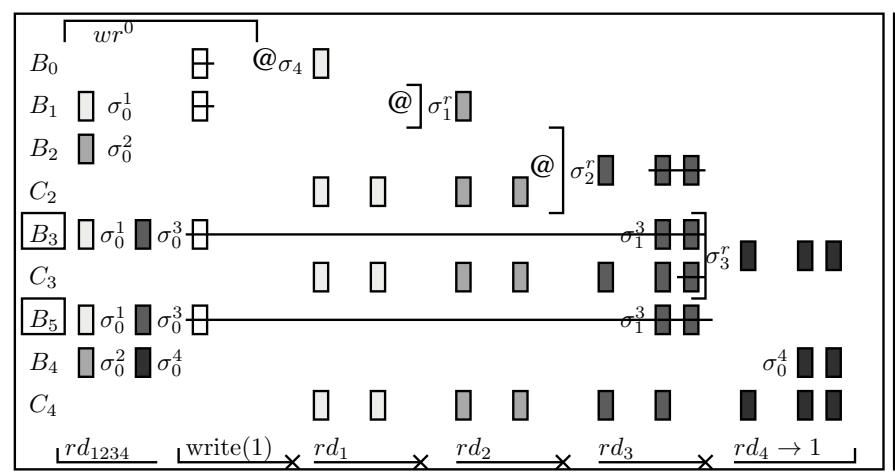

(g) $p r_{4}$ extends $\Delta p r_{3}\left(\Delta p r_{4}\right.$ from $\left.p r_{4}\right)$ by deleting crossed steps

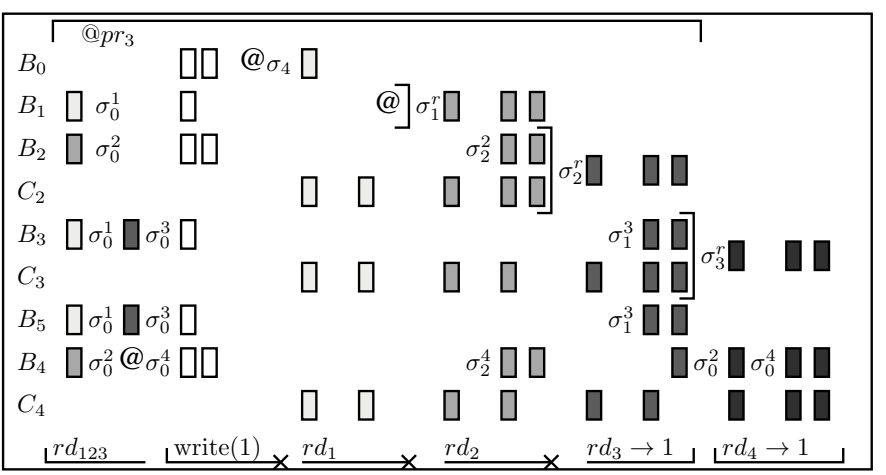

(h) $p r_{4}^{C}$ (extends @ $r_{3} \sim p r_{3}$ )

Figure 2: Instance of the proof with $k=4$.

Next, we define partial run $\Delta p r_{1}$ obtained from $p r_{1}$ by deleting the steps of the read and the write as illustrated in Figure 2 (a). More specifically, $\Delta p r_{1}$ extends $w r^{k-2}$ by appending the missing steps of an incomplete read $r d_{1}$ of type inc3, after which $r d_{1}$ crashes. In $\Delta p r_{1}, \mathcal{M}_{0}=\left\{B_{0}\right\}$ is malicious and forges its state to $\sigma_{k}$ before replying to $r d_{1}$.
Observe that at the end of $p r_{1}$ and $\Delta p r_{1}$, every correct block is in the same state, except $\mathcal{P}_{2}$. We refer to the state of $B_{1}$ at the end of $\Delta p r_{1}$ as $\sigma_{1}^{r}$.

Starting from $\Delta p r_{1}$ we iteratively define the following partial runs for $2 \leq l \leq k$ (see Fig. 2). Partial run $p r_{l}$ extends $\Delta p r_{l-1}$ by appending the missing steps of a complete 
read $r d_{l}$. In $p r_{l}$, superblock $\mathcal{M}_{l-2}$ is malicious and all other blocks are correct. Since $r d_{l}$ does not receive any messages from $\mathcal{M}_{l-2}$, it completes only on the basis of replies from correct objects (at least $S-t_{k}$ by equation (1)). At the end of $p r_{l}, r d_{l}$ completes and returns value $x$. To show that $x=1$, we define a partial run @ $p r_{l-1}$ which is identical to $p r_{l-1}$ except that in @ $p r_{l-1}$ (1) there is no read by $r_{l}$ and (2) and (in addition to $\mathcal{M}_{l-3}$ ), superblock $\mathcal{P}_{l}$ is malicious and forges its state to $\sigma_{0}^{l}$, simulating the occurrence of $r d_{l}$ as in $p r_{l-1}$. The count of malicious objects in @ $p r_{l-1}$ is exactly $t_{k}$. To see why, notice that by equation (1) and (2) the malicious objects in @ $p r_{l-1}$ amount to $\left|\bigcup \mathcal{P}_{l}\right|+\left|\bigcup \mathcal{M}_{l-3}\right|=t_{k}-t_{l-2}+t_{l-2}=t_{k}$.

Then, partial run $p r_{l}^{C}$ extends @ $p r_{l-1}$ by appending $r d_{l}$. Note that $r d_{l}$ cannot distinguish $p r_{l}^{C}$ from $p r_{l}$ because superblock $\mathcal{P}_{l}$, which is malicious in $p r_{l}^{C}$, mimics $p r_{l}$. In particular, $\mathcal{P}_{l}$ forges its state to $\sigma_{0}$ before replying to $r d_{l}$ 's first round and then to $\sigma_{k-l}^{*}$ before replying to $r d_{l}$ 's second round. By atomicity, $r d_{l}$ returns 1 in $p r_{l}^{C}$. Since $p r_{l}^{C}$ and $p r_{l}$ are indistinguishable to reader $r_{l}, x=1$.

Next, we define partial run $\Delta p r_{l}$. For $2 \leq l<k, \Delta p r_{l}$ is obtained from $p r_{l}$ by deleting steps of $r d_{l}, r d_{l-1}$ and the write (see Fig. 2 (c) and (e)). In $\Delta p r_{l}$, superblock $\mathcal{M}_{l-1}$ is malicious, all other block are correct, and blocks $\left\{B_{l-1}\right.$, $\left.C_{l-1}\right\} \in \mathcal{M}_{l-1}$ forge their state to $\sigma_{j}^{r}$ before replying to $r d_{l} .^{5}$ In more detail, $\Delta p r_{l}$ extends $w r^{k-l-1}$ by appending the missing steps (1) of incomplete reads $r d_{1}, \ldots, r d_{l-1}$ of type inc2, and (2) of an incomplete $r d_{l}$ of type inc3. $B_{0}$ forges its state to $\sigma_{k}$ before replying to $r d_{1}$ and for $1 \leq$ $j \leq l-1,\left\{B_{j}, C_{j}\right\}$ forge their state to $\sigma_{j}^{r}$ before replying to $r d_{j+1}$. Observe that at the end of $p r_{l}$ and $\Delta p r_{l}$, every correct block is in the same state, except $\mathcal{P}_{l+1}$. We refer to the state of $\left\{B_{l}, C_{l}\right\}$ at the end of $\Delta p r_{1}$ as $\sigma_{l}^{r}$.

Finally, partial run $\Delta p r_{k}$ is obtained analogously from $p r_{k}$, except that in $\Delta p r_{k}$, (a) no write is invoked and (b) read $r d_{k}$ is complete and skips $\mathcal{M}_{k-2} \cup \mathcal{P}_{k+1}$ (see Fig. 2 (g) for $k=4)$. In particular, in $\Delta p r_{k}, \mathcal{M}_{k-1}$ is malicious and blocks $\left\{B_{k-1}, C_{k-1}\right\} \in \mathcal{M}_{k-1}$ forge their state to $\sigma_{k-1}^{r}$ before replying to $r d_{k}$. By equation (1) the malicious objects amount to $\left|\bigcup \mathcal{M}_{k-1}\right|=t_{k}$. Partial runs $p r_{k}$ and $\Delta p r_{k}$ differ only at $\mathcal{P}_{k+1}$, and $r d_{k}$ completes without receiving any message from $\mathcal{P}_{k+1}$. Thus, $r d_{k}$ cannot distinguish $\Delta p r_{k}$ from $p r_{k}$ and returns 1 in $\Delta p r_{k}$, a contradiction, as no write was invoked.

LEMmA 2. : If $S \leq 3 t+1$ and every read completes in three rounds then no write implementation $I$ of a multireader $(S W M R)$ atomic register exists that completes in $\min \{R,\lfloor\log (\lceil(3 t+1) / 2\rceil)\rfloor\}$ rounds.

Proof. Let $k=\min \{R,\lfloor\log (\lceil(3 t+1) / 2\rceil)\rfloor\}$, i.e., $R \geq k$ and $k \leq\lfloor\log (\lceil(3 t+1) / 2\rceil)\rfloor$. By Lemma 1 , there exists no optimally resilient $k$-reader atomic register implementation with $t_{k}=t_{k-1}+2 t_{k-2}+1$ faulty objects, where the read completes in three rounds and the write completes in $k$ rounds. Observe that this is valid even with $R \geq k$ readers and $t \geq t_{k}$ faults. Writing $t_{k}$ in closed form results in $t_{k}=\frac{1}{6}\left(2^{k+2}-(-1)^{k}-3\right)$. Thus, we have that $t \geq \frac{1}{6}\left(2^{k+2}-(-1)^{k}-3\right)$. Solving for $k$ results in $k \leq$ $\lfloor\log (\lceil(3 t+1) / 2\rceil)\rfloor$.

\footnotetext{
${ }^{5}$ The states are different and are indexed by the object's id, which for simplicity of presentation is made implicit.
}

Finally, we generalize our result to a resilience of $3 t+$ $\left\lfloor t / t_{k}\right\rfloor$ for $t \geq t_{k}$, proving Proposition 2 .

PROOF. Without loss of generality we can assume that $t \geq t_{k}$ because every implementation is subject to the resilience lower bound of $S \geq 3 t+1$. The observation is that if we multiply each of the blocks in the proof of Lemma 1 with a constant $c$, then the result holds for $S^{\prime}=c S=3 c t_{k}+c$ objects and $c t_{k}$ faults. By carefully choosing $c=t / t_{k}$, we obtain a lower bound proof for $S^{\prime}=3 t+\left\lfloor t / t_{k}\right\rfloor$ and $t$ faults.

\section{CONCLUSION}

In this paper, we show that no single-writer multiplereader (SWMR) robust atomic storage implementation exists if (a) read operations complete in less than four communication round-trips (rounds), and (b) the time-complexity of write operations is constant.

However, we observe that a matching implementation can simply be obtained by a) reusing the SWMR regular storage implementation of [15] which features the worst-case time complexity of 2 rounds for both reads and writes, and b) transforming it to the SWMR atomic implementations using a standard SWMR regular - SWMR atomic transformation technique $[4,20] .{ }^{6}$ This yields a sought SWMR atomic implementation in which write operations complete in 2 rounds whereas reads complete in 4 rounds.

Furthermore, in the stronger authentication model that allows for secret values [8], regular storage of [15] can be replaced in the above transformation with the corresponding time-optimal regular implementation [8], yielding a 2-round write 3-round read atomic storage, which is optimal in this model. In both models, multi-writer atomic storage can be implemented by applying the standard transformations further $[4,20]$.

In summary, we present two lower bounds. The first is a read lower bound stating that three rounds of communication are necessary to read from a SWMR robust atomic storage. The second is a write lower bound, showing that $\Omega(\log (t))$ write rounds are necessary to read in three rounds from such a storage. Our results close a fundamental gap: we show that time-optimal, 2-round write 4-round read (resp. 3 -round read in the secret value model) robust atomic storage can be obtained using well-known transformations from regular to atomic storage and existing time-optimal regular storage implementations.

\section{REFERENCES}

[1] Ittai Abraham, Gregory Chockler, Idit Keidar, and Dahlia Malkhi. Byzantine disk paxos: optimal resilience with byzantine shared memory. Distributed Computing, 18(5):387-408, 2006.

[2] Amitanand S. Aiyer, Lorenzo Alvisi, and Rida A. Bazzi. Bounded wait-free implementation of optimally resilient byzantine storage without (unproven) cryptographic assumptions. In Proceedings of the 21st International Symposium on Distributed Computing, pages 7-19, September 2007.

\footnotetext{
${ }^{6}$ In short, this transformation employs $R+1$ regular registers, one dedicated to the writer and $R$ additional ones, one per reader, in which a given reader writes back the read value.
} 
[3] Hagit Attiya, Amotz Bar-Noy, and Danny Dolev. Sharing memory robustly in message-passing systems. Journal of the ACM, 42(1):124-142, 1995.

[4] Hagit Attiya and Jennifer Welch. Distributed Computing. Fundamentals, Simulations, and Advanced Topics. McGraw-Hill, 1998.

[5] AWS Simple Storage Service. http://aws.amazon.com/s3/.

[6] Christian Cachin, Idit Keidar, and Alexander Shraer. Trusting the cloud. SIGACT News, 40(2):81-86, 2009.

[7] Dan Dobre, Rachid Guerraoui, Matthias Majuntke, Neeraj Suri, and Marko Vukolić. The Complexity of Robust Atomic Storage. Technical Report TR-TUD-DEEDS-06-01-2010, 2010.

[8] Dan Dobre, Matthias Majuntke, Marco Serafini, and Neeraj Suri. Efficient robust storage using secret tokens. In Proceedings of the 11th International Symposium on Stabilization, Safety, and Security of Distributed Systems, pages 269-283, 2009.

[9] Partha Dutta, Rachid Guerraoui, Ron R. Levy, and Arindam Chakraborty. How fast can a distributed atomic read be? In Proceedings of the 23rd annual ACM symposium on Principles of distributed computing, pages 236-245, July 2004.

[10] Partha Dutta, Rachid Guerraoui, Ron R. Levy, and Marko Vukolic. Fast access to distributed atomic memory. SIAM J. Comput., 39(8):3752-3783, 2010.

[11] Burkhard Englert, Chryssis Georgiou, Peter M. Musial, Nicolas C. Nicolaou, and Alexander A. Shvartsman. On the efficiency of atomic multi-reader, multi-writer distributed memory. In Proceedings of the 13th International Conference on Principles of Distributed Systems, pages 240-254, 2009.

[12] Rui Fan and Nancy Lynch. Efficient replication of large data objects. In Proceedings of the 17th International Symposium on Distributed Computing, pages 75-91, October 2003.

[13] Chryssis Georgiou, Nicolas C. Nicolaou, and Alexander A. Shvartsman. Fault-tolerant semifast implementations of atomic read/write registers. $J$. Parallel Distrib. Comput., 69(1):62-79, 2009.

[14] Rachid Guerraoui, Ron R. Levy, and Marko Vukolić. Lucky read/write access to robust atomic storage. In Proceedings of the International Conference on Dependable Systems and Networks, pages 125-136, 2006.

[15] Rachid Guerraoui and Marko Vukolić. How fast can a very robust read be? In Proceedings of the twenty-fifth annual ACM symposium on Principles of distributed computing, pages 248-257, New York, NY, USA, 2006. ACM.

[16] Rachid Guerraoui and Marko Vukolić. Refined quorum systems. In Proceedings of the twenty-sixth annual ACM symposium on Principles of distributed computing, pages 119-128, 2007.

[17] Maurice Herlihy. Wait-free synchronization. ACM Transactions on Programming Languages and Systems, 13(1):124-149, January 1991.

[18] Leslie Lamport. On interprocess communication. Distributed computing, 1(1):77-101, May 1986.

[19] Leslie Lamport, Robert E. Shostak, and Marshall C.
Pease. The Byzantine generals problem. ACM Transactions on Programming Languages and Systems, 4(3):382-401, 1982.

[20] Nancy A. Lynch. Distributed Algorithms. Morgan-Kaufmann, 1996.

[21] Nancy A. Lynch and Mark R.Tuttle. An introduction to input/output automata. CWI Quarterly, 2(3):219-246, 1989.

[22] Nancy A. Lynch and Alexander A. Shvartsman. Rambo: A reconfigurable atomic memory service for dynamic networks. In Proceedings of the 16th International Conference on Distributed Computing, pages 173-190, London, UK, 2002. Springer-Verlag.

[23] Jean-Philippe Martin, Lorenzo Alvisi, and Michael Dahlin. Minimal Byzantine storage. In Proceedings of the 16th International Conference on Distributed Computing, pages 311-325, October 2002.

[24] Yasushi Saito, Svend Frolund, Alistair Veitch, Arif Merchant, and Susan Spence. Fab: building distributed enterprise disk arrays from commodity components. SIGOPS Oper. Syst. Rev., 38(5):48-58, 2004.

[25] Frank Schmuck and Roger Haskin. GPFS: A shared-disk file system for large computing clusters. In Proceedings of the 1st USENIX Conference on File and Storage Technologies, pages 231-244, Berkeley, CA, USA, 2002. USENIX Association.

[26] Philip M. Thambidurai and You-Keun Park. Interactive consistency with multiple failure modes. In Symposium on Reliable Distributed Systems, pages 93-100, 1988. 